\title{
Enxertos ósseos em odontologia - uma revisão integrativa da literatura
}

\author{
Bone grafts in dentistry - an integrative literature review \\ Injertos de hueso en odontología - revisión integrativa de la literatura
}

Recebido: 22/09/2021 | Revisado: 27/09/2021 | Aceito: 27/09/2021 | Publicado: 29/09/2021

Lucas Menezes dos Anjos

ORCID: https://orcid.org/0000-0001-5100-0789

Universidade Federal de Santa Catarina, Brasil

E-mail: luks_anjos@hotmail.com

Aurélio de Oliveira Rocha

ORCID: https://orcid.org/0000-0002-9308-2118

Universidade Federal de Santa Catarina, Brasil

E-mail: aureliorocha2015@gmail.com

Thaine Oliveira Lima

ORCID: https://orcid.org/0000-0001-5220-9947

Universidade de São Paulo, Brasil

E-mail: thaineol95@gmail.com

Rafaela de Menezes dos Anjos Santos

ORCID: https://orcid.org/0000-0002-1517-4527 Universidade Federal de Sergipe, Brasil E-mail: anjosrafa@outlook.com

Maria de Nazaré Oliveira Rocha

ORCID: https://orcid.org/0000-0002-2655-8083

Faculdade Maurício de Nassau, Brasil E-mail: mariaoli2017@outlook.com.br

Nailson Silva Meneses Júnior

ORCID: https://orcid.org/0000-0003-1384-810X

Universidade de São Paulo, Brasil

E-mail: menesesnailson@gmail.com

Maraiza Alves de Oliveira

ORCID: https://orcid.org/0000-0002-1674-6451 Universidade Federal de Sergipe, Brasil

E-mail: mara.iza7889@gmail.com

Marlon Vinícius Santos Reis

ORCID: https://orcid.org/0000-0002-1580-2124 Universidade Federal de Sergipe, Brasil

E-mail: marlonvinicius.sr@live.com Aparecida Emanoelly Sales de Melo ORCID: https://orcid.org/0000-0002-5579-3998 Universidade Tiradentes, Brasil E-mail:emanoellysales@hotmail.com

Pablo Jordão Alcântara Cruz

ORCID: https://orcid.org/0000-0002-0235-692X Universidade Federal de Sergipe, Brasil

E-mail:pablo.jordao@hotmail.com

\begin{abstract}
Resumo
A exodontia de unidades permanentes, assim como doença periodontal e trauma bucal, estão muitas vezes relacionados ao processo de reabsorção alveolar, o que culminam na perda óssea tanto em espessura quanto em altura, sendo esse principal impasse para reabilitação oral por meio de prótese sobre implantes. Cerca de 50\% dos sítios para instalação de implantes não apresentam volume ósseo suficiente para sua instalação, o leva a necessidade de utilizar biomateriais para o processo de regeneração óssea guiada. Diante da necessidade de aumentar tecido ósseo em volume e espessura, diversos biomateriais foram desenvolvidos, sendo eles classificados em enxertos do tipo autógeno, alógeno, xenógeno e aloplástico. Para realização desse estudo foi realizada uma busca completa nas bases de dados: PubMed, Scielo, Lilacs e Google Scholar, utilizando as palavras-chave "dentistry bone transplantation, surgery oral, biocompatible materials and bone regeneration", inseridas nas buscas de maneira cruzada adotando a expressão boolena "and". Afim de selecionar os artigos a serem analisados foram elencados critérios de inclusão e exclusão. A utilização de enxertos ósseos tem mostrado resultados promissores no ganho de osso vertical e horizontal. A
\end{abstract}


associação de biomaterias permite que as melhores propriedades de cada tipo de enxerto sejam usadas de forma integrada, o que leva a resultados favoráveis tanto histologicamente como funcionalmente.

Palavras-chave: Transplante Ósseo; Cirurgia Oral; Materiais Biocompatíveis e Regeneração Óssea.

\begin{abstract}
The extraction of permanent units, as well as periodontal disease and oral trauma, are often related to the alveolar resorption process, which culminates in bone loss both in terms of thickness and height, which is the main impasse for oral rehabilitation through prosthesis over implants. About 50\% of the sites for implant installation do not have enough bone volume for their installation, leading to the need to use biomaterials for the guided bone regeneration process. Given the need for bone increase in volume and thickness, several biomaterials were developed, which were classified as autogenous, allogeneic, xenogenous and alloplastic grafts. To carry out this study, a complete search was carried out in the following databases: PubMed, Scielo, Lilacs and Google Scholar, using as keywords "dental bone transplantation, oral surgery, biocompatible materials and bone regeneration", inserted in the searches crosswise, adopting the Boolean expression "e". In order to select the articles to be promoted, inclusion and exclusion criteria were listed. The use of bone grafts has promising results in vertical and horizontal bone gain. The association of biomaterials allows the best properties of each graft type are used in an integrated way, which leads to favorable results both histologically and functionally.
\end{abstract}

Keywords: Bone Transplant; Oral Surgery; Biocompatible Materials and Bone Regeneration.

\title{
Resumen
}

La extracción de unidades permanentes, así como la enfermedad periodontal y el traumatismo bucal, suelen estar relacionadas con el proceso de reabsorción alveolar, que culmina en la pérdida ósea tanto en grosor como en altura, que es el principal callejón sin salida de la rehabilitación oral mediante prótesis sobre implantes. Aproximadamente el $50 \%$ de los sitios para la instalación de implantes no tienen suficiente volumen óseo para su instalación, lo que lleva a la necesidad de utilizar biomateriales para el proceso de regeneración ósea guiada. Ante la necesidad de incrementar el tejido óseo en volumen y grosor, se desarrollaron varios biomateriales, que fueron clasificados como injertos autógenos, alogénicos, xenogénicos y aloplásticos. Para la realización de este estudio se realizó una búsqueda completa en las siguientes bases de datos: PubMed, Scielo, Lilacs y Google Scholar, utilizando las palabras clave "odontología trasplante óseo, cirugía oral, materiales biocompatibles y regeneración ósea", insertadas en las búsquedas de forma transversal, adoptando la expresión booleana "y". Para la selección de los artículos a analizar se enumeraron los criterios de inclusión y exclusión. El uso de injertos óseos ha mostrado resultados prometedores en la ganancia ósea vertical y horizontal. La asociación de biomateriales permite obtener las mejores propiedades de cada tipo de injerto se utiliza de forma integrada, lo que conduce a resultados favorables tanto histológica como funcionalmente.

Palabras clave: Trasplante Óseo; Cirugía Oral; Materiales Biocompatibles y Regeneración Ósea.

\section{Introdução}

A exodontia de unidades permanentes, assim como doença periodontal e trauma bucal estão muitas vezes relacionados ao processo de reabsorção alveolar, o que culminam na perda óssea tanto em espessura quanto em altura. A perda do osso alveolar tem sido o principal impasse para reabilitação oral por meio de prótese sobre implantes (Nadja et al, 2019).

O crescente número de pesquisa sobre implantes dentários osseointegrados e a popularização do seu uso têm levado ao aumento da procura por procedimentos para reconstrução do osso alveolar (Lima et al, 2018). Segundo Bosshart e Schenk (2010) cerca de 50\% dos sítios para instalação de implantes não apresentam volume ósseo suficiente para realização do procedimento. A quantidade suficiente de osso permite a instalação de implantes funcionalmente e esteticamente bemsucedidos, e para que se consiga chegar a resultados satisfatórios muitas vezes o cirurgião-dentista precisa lançar mão de técnicas de regeneração óssea (Adel e Marwa, 2018).

As opções de biomateriais disponíveis para reconstrução óssea na odontologia atualmente são enxerto ósseo autógeno, tendo como doador o próprio individuo; alógeno, proveniente de indivíduos da mesma espécie, porém geneticamente diferentes; xenógeno, o qual deriva de outra espécie animal; e por fim os biomateriais produzidos de forma sintética em laboratórios, os chamados enxertos aloplásticos (Klijn et al, 2010; Troeltzsch et al, 2016). 
O osso autógeno é o único substituído ósseo que apresenta as propriedades ideais de um biomaterial, sendo elas a osteogênese, osteocondução e osteindução. A osteogênese compreende a formação óssea por meio de células osteoprogenitoras que se diferenciam em osteoblastos levando a produção ativa de osso. O processo de osteindução induz célulastronco mesenquimais a se diferenciarem em células de linhagem osteogênicas por influência de proteínas indutoras ou morfogênicas do osso, fatores de crescimento ou citocinas. Já para que haja osteocondução é necessário um arcabouço para que seja desenvolvido um novo tecido ósseo, sendo a matriz reabsorvida e substituída por osso (Julic e Perciaccante, 2004; Giannoudis et al, 2005).

Mesmo o osso autógeno sendo considerado a melhor opção ao levar em consideração suas propriedades biológicas, as técnicas cirúrgicas necessárias para captação desse tipo de enxerto aumentam a morbidade do procedimento e expõem o paciente à complicações como infecção, perda sanguínea, lesão nervosa, deficiência funcional e dor no pós-operatório (Kalk et al, 1996; Raghoebar et al, 2007). Outras desvantagens desse tipo de técnica são a necessidade de abordar dos dois sítios cirúrgicos, oferta limitada de osso, altos custos e maior índice de reabsorção (Cristiane et al, 2013).

Nas regenerações ósseas guiadas, diferentes biomateriais têm sido associados ao enxerto autógeno com o intuito de minimizar a morbidade associada ao segundo sítio cirúrgico e de diminuir a taxa de reabsorção do osso autógeno (Urban, 2009). O presente estudo tem como objetivo realizar uma revisão integrativa da literatura abordando o uso dos diferentes materiais de enxerto ósseo na odontologia.

\section{Metodologia}

Realizou-se uma revisão integrativa, qualitativa, de cunho descritivo por meio de pesquisa bibliográfica exploratória, nas bases de dados PubMed (www.pubmed.com), Scielo (www.scielo.com), GoogleScholar (scolar.google.com.br) e Lilacs (www.lilacs.com). A pesquisa foi realizada no mês de setembro de 2021, utilizando os descritores "Dentistry Bone Transplantation, Surgery Oral, Biocompatible Materials and Bone Regeneration” os quais foram inseridos na plataforma de maneira cruzada adotando a expressão booleana "and". Após a seleção dos artigos, de acordo com os critérios de inclusão e exclusão descritos na Tabela 1, os mesmos foram fichados e tabulados (Souza e Carvalho, 2010).

Tabela 1 - Tabela com os critérios de inclusão e exclusão elencados para seleção dos artigos.

\begin{tabular}{clll}
\hline & Artigos científicos publicados & & \\
CRITÉRIOS DE & em revistas nacionais ou & Período: setembro de 2016 a & Idiomas: Inglês, espanhol e \\
INCLUSÃO. & internacionais, que & setembro de 2021. & português \\
& abrangessem os descritores & & \\
& propostos. & & \\
\hline CRITÉRIOS DE & Revisão de literatura que não & Texto: não disponível & Outros Idiomas \\
EXCLUSÃO. & tratasse do objetivo proposto & \\
& & & \\
\hline
\end{tabular}

Fonte: Anjos LM, et al., (2021).

Pode ser observado na Tabela 1 os critérios de inclusão (tipo de estudo, período de publicação e idioma) e exclusão (tipo de estudo e idioma) pré determinados para inclusão dos artigos selecionados para análise. 


\section{Resultados e Discussão}

\section{Tecido Ósseo}

O osso é um dos tecidos mais resistentes do copo humano, e apesar de ter um alto grau de rigidez, apresenta certo grau de elasticidade, propriedade essa que o torna resistente a forças do tipo tração e compressão (Nadja et al, 2019). Sua rigidez se dá pela presença de matriz inorgânica, que corresponde cerca de $67 \%$ de sua composição, sendo formada em sua maioria por hidroxiapatita. Já a matriz orgânica, correspondente a 33\%, e composta principalmente por colágeno do tipo I (Kunz et al, 2017).

Por ser um tecido vivo, apresenta células que garantes sua formação, remodelação e reparo, sendo elas os osteoblastos, osteoclastos, osteócitos e as células osteoprogenitoras. Os osteoblastos são responsáveis pela deposição do tecido ósseo, estando vinculados ao processo de formação óssea, assim como no reparo. Os osteoclastos são células gigantes que tem como função reabsorver a matriz orgânica óssea, participando de forma ativa do processo de remodelação do osso. Os osteócitos se formam a partir da maturação dos osteoblastos, quando os mesmos são envoltos por matriz orgânica formando um emaranhado ligadas por prolongamentos (Azambuja et al, 2019). Já as células osteoprogenitoras são consideras células de reserva óssea, as quais são acionadas quando há um processo de injuria tecidual e há necessidade de reparação. Na presença de tecidual injuria tecidual, as células osteoprogenitoras se diferenciam em osteoblastos, os quais depositam matriz óssea no local que necessita de reparo (Kunz et al, 2017).

O tecido ósseo tem como função principal a de proteger órgãos vitais como coração, pulmão e massa cefálica, assim como, promover a sustentação e movimentação do corpo humano dando suporte a musculatura e permitindo seu deslize por meio das articulações; é uma reversa de cálcio; e aloja a medula óssea (Haugen et al, 2019).

\section{Regeneração Óssea Guiada}

A reabsorção alveolar é um processo de remodelação óssea que inicia rapidamente após a realização de exodontias, assim como em situações de traumas dentoalveolares e doença periodontal crônica. Um rebordo alveolar reabsorvido reduz de forma significativa a possibilidade de inserção de implantes ósseo integrados (Wang e Carroll, 2004).

Diante da presença de um rebordo alveolar reabsorvido que necessite ser reabilitado por meio de implantes dentais ósseo integrados, o cirurgião-dentista pode lançar mão a técnica de regeneração óssea guiada (ROG) para promover ganho ósseo regional e posterior instalação de implantes. Essa técnica é baseada na osteopromoção, que se refere ao uso de biomateriais para promover um selamento total de um local anatômico e prevenir que outros tecidos, principalmente tecido conjuntivo, não interfiram na osteogênese, bem como no direcionamento da formação óssea (Acevedo et al, 2004).

Alguns requisitos são necessários para que se consiga resultados previsíveis na técnica de ROG, sendo eles: fonte de células osteogênicas, osso viável e livre de infecção, vascularização abundante, local da ferida cirúrgica mecanicamente estável durante o processo de cicatrização e espaço adequado para deposição de biomateriais (Rodolfo et al, 2017).

\section{Tipos de enxertos ósseo}

Segundo Lima et al. (2018), os biomateriais são substâncias ou combinação de sustâncias de natureza sintética ou natural utilizados para aumentar ou substituir parcialmente ou integralmente tecidos e órgãos. Em odontologia, os enxertos ósseos são os biomateriais mais utilizados, tendo por função facilitar o povoamento celular e o crescimento de tecido na área defeituosa (Pilger et al, 2020).

Os biomateriais podem apresentar três propriedades básicas, sendo elas a osteogênese, a osteoindução e a osteocondutoção. A osteogênese se refere a enxertos orgânicos que são capazes de estimular a formação de osso diretamente a partir de osteoblastos, ou seja, são enxertos que carregam com si os osteoblastos, que são células de formação óssea (Urist et 
al, 1984). A osteoindução é a propriedade dos biomateriais induzir a diferenciação de células mesenquimais, ou células indiferenciadas em osteoblastos ou condroblastos, aumentando a formação óssea no local ou mesmo estimular a formação de osso em um sítio heterotópico. Já a ostecondução, propriedade geralmente relacionada a enxertos do tipo inorgânico, permite a aposição de um novo tecido ósseo na sua superfície, requerendo a presença de tecido ósseo pré-existente como fonte de células osteoprogenitoras (Urist et al, 1984; Rodolfo et al, 2017).

O enxerto ósseo autógeno é considerado o único material que apresenta as três propriedades ideias dos biomateriais osteogênese, osteindição e osteocondição-, sendo considerado por muito tempo o padrão ouro dos substitutos ósseos na regeneração óssea guiada (Ferreira Filho et al, 2021). Conduto, a necessidade de um segundo momento cirúrgico para coleta do osso autógeno é sua principal limitação, pois em pacientes sistêmicos essa pode ser uma contraindicação para sua utilização. A baixa disponibilidade de osso na área doadora, a comorbidade causada pelo procedimento cirúrgico e o alto índice de reabsorção quando instalado no sitio receptor, também são limitações para sua utilização (Soni et al, 2019).

Buscando minimizar as limitações na realização da técnica com uso do enxerto autógeno, outras alternativas foram desenvolvidas, como o enxerto alógeno, xenógeno e os biomateriais produzidos em laboratórios, os chamados enxertos aloplásticos (Wen et al, 2019).

Em 1988, Masters já relatava os benefícios do enxerto ósseo alógeno, o qual é obtido a partir de doadores da mesma espécie com carga genotípica diferente. O autor afirma que por ser tratado sob congelamento profundo em temperaturas inferiores a $-60^{\circ} \mathrm{C}$, sua degradação é diminuída, assim como a possibilidade de desenvolvimento de resposta imunológica no paciente receptor. Segundo Urban (2009), o aloenxerto mineralizado apresenta boa propriedade osteocondutora, pois sua estrutura altamente porosa mantém sua altura, o que favorece a regeneração tecidual, além de permitir melhor molhabilidade do material por sangue. Já o aloenxerto desmineralizado apresenta baixa ostocondutibilidade e alto grau de reabsorção (Naenni, et al, 2019). Contudo, estudos com resultados a longo prazo ainda são necessários para demostrar os níveis de reabsorção ou desenvolvimento de resposta imune no paciente receptor.

Outra alternativa para reconstruções ósseas previas a instalação de implantes, são os enxertos ósseos do tipo xenógeno, os quais são obtidos a partir animais (Sampaio et al, 2020). O osso bovino medular esterializado e desproteinizado é o xenoenxeto mais utilizado e documentado na literatura, pois consegue se incorporar bem ao leito receptor devido sua topografia superficial, o que permite excelente interação com o coagulo sanguíneo, favorecendo a proliferação vascular e celular (Fardin et al, 2010). Por ser reabsorvido lentamente, consegue manter o arcabouço necessário para ostecondução (Fardin et al, 2010). Devido seu baixo índice de reabsorção, frequentemente é utilizado em associação com o enxerto autógeno, o que faz com que o biomaterial fique por mais tempo no leito receptor, situação que permite respeitar o tempo necessário para que se alcance a regeneração óssea sem que o enxerto seja reabsorvido (Haugen et al, 2019).

Finalmente, os enxertos ou substitutos aloplásticos são biomateriais puramente sintéticos produzidos em laboratório, mas que carregam consigo componentes químicos essenciais do tecido ósseo natural, como o cálcio e fosforo, sendo estes essenciais para regeneração óssea (Haugen et al, 2019). As vantagens comuns dos substitutos ósseos aloplásticos são a qualidade do produto padronizado, e a ausência de risco de doenças infecciosas e disponibilidade ilimitada de material (Stevenson et al, 1993; Hsu e Wang, 2013). Por apresentarem baixa capacidade regenerativa, os substitutos ósseos aloplásticos são frequentemente usados em associação a outras classes de enxerto ósseo, assim como aplicados com fatores de crescimento e / ou membranas (Hsu e Wang, 201; Haugen et al, 2019; Armitage, 2020). 


\section{Conclusão}

A utilização de enxertos ósseos tem mostrado excelentes resultados no ganho de osso vertical e horizontal. A correta anamnese, leitura dos exames de imagem para verificação da necessidade ou não de enxerto ósseo, a realização de uma técnica cirúrgica minuciosa, assim como a correta indicação do tipo de enxerto a ser usado, são fatores essenciais para o processo de regeneração óssea e promissora reabilitação por implantes ósseos integrados.

Ademais, diante do crescente número de biomateriais sendo desenvolvidos, é fundamental o ampliamento de mais pesquisas que possam mostrar resultados a longo prazo.

\section{Referências}

Acevedo, R., Trentin, M. S., Shibli, J. A., \& Marcantonio Jr, E. (2004). Bases clínicas e biológicas da regeneração óssea guiada (ROG) associada a barreiras ou membranas. Rev Bras Implantodont Prótese Implant, 11(43), 251-7.

Adel, S. A. e Marwa, M. A. D. I (2018). Localized ridge augmentation in the anterior maxilla using titanium mesh, an alloplast, and a nano-bone graft: a case repor. Journal of International Medical Research, Saudi Arabia, 2001-2007.

Armitage, G. C. (2020). A brief history of periodontics in the United States of America: Pioneers and thought-leaders of the past, and current challenges. Periodontology 2000, 82(1), 12-25.

Azambuja Carvalho, P. H., dos Santos Trento, G., Moura, L. B., Cunha, G., Gabrielli, M. A. C., \& Pereira-Filho, V. A. (2019). Horizontal ridge augmentation using xenogenous bone graft-systematic review. Oral and maxillofacial surgery, 23(3), 271-279.

Ferreira Filho, M. J. S., Miranda, T. T., Barros, D. N. R., Pavane, R. M., da Silva Pimenta, Y., da Silva Mousinho, L., \& Milério, L. R. (2021). Enxerto autógeno em bloco em região de pré maxila: relato de caso. Brazilian Journal of Development, 7(1), 591-603.

Bosshart, D. D., \& schenk, R. K. (2010). Base biológica da regeneração óssea. In: 20 anos de regeneração óssea guiada na implantodontia. São Paulo: Quintessence. Cap.2. p. 15-45.

Fardin, A. C., Jardim, E. C. G., Pereira, F. C., Guskuma, M. H., Aranega, A. M., \& Garcia Júnior, I. R. (2010). Enxerto ósseo em odontologia: revisão de literatura. Innovations Implant Journal, 5(3), 48-52.

Giannoudis, P. V., Dinopoulos, H., Tsiridis, E (2005). Bone substitutes: an update. Injury, (3):20-7.

Haugen, H. J., Lyngstadaas, S. P., Rossi, F., \& Perale, G. (2019). Bone grafts: which is the ideal biomaterial?. Journal of Clinical Periodontology, 46, 92-102.

Hsu, Y. T., \& Wang, H. L. (2013). How to select replacement grafts for various periodontal and implant indications. Clinical Advances in Periodontics, 3(3), $167-179$.

Kalk, W. W., Raghoebar, G. M., Jansma, J., Boering, G. (1996). Morbidity from iliac crest bone harvesting. J Oral Maxillofac Surg, 54(12):1424-1430.

Klijn, R. J., Meijer, G. J., Bronkhorst, E. M., Jansen, J. A (2010). A meta-analysis of histomorphometric results and graft healing time of various biomaterials compared to autologous bone used as sinus floor augmentation material in humans. Tissue Eng Part B Ver, 16(5):493-507.

Kunz, R. I., Natali, M. R. M., Torquato, E. F. B., Ribeiro, L. D. F. C., Della Justina, L. A., \& Brancalhão, R. M. C. (2017). Proposta didática no ensino integrado da morfologia: células e tecido ósseo. Experiências em Ensino de Ciências, 12(2), 38-52

Lima, J. L. O, Sendyk, D. I., Sendyk, W. R., Polo, C. I., Correa, L., Deboni, M. C. Z (2018). Growth Dynamic of Allogeneic and Autogenous Bone Grafts in a Vertical Model. Braz Dent J., 29(4):325-334.

Masters, D. H. (1988). Implants. Bone and bone substitutes. CDA journal, 16(1), 56-65.

Naenni, N., Bienz, S. P., Jung, R. E., Hämmerle, C. H., \& Thoma, D. S. (2019). Histologic analyses of flapless ridge preservation in sockets with buccal dehiscence defects using two alloplastic bone graft substitutes. Clinical oral investigations, 23(9), 3589-3599.

Polo, C. I., Lima, J. L. O., De Lucca, L., Piacezzi, C. B., Naclério-Homem, M. D. G., Arana-Chavez, V. E., \& Sendyk, W. R. (2013). Effect of recombinant human bone morphogenetic protein 2 associated with a variety of bone substitutes on vertical guided bone regeneration in rabbit calvarium. Journal of periodontology, 84(3), 360-370.

Pilger, A. D. A., Schneider, L. E., da Silva, G. M., Schneider, K. C. C., \& Smidt, R. (2020). Membranas e barreiras para regeneração óssea guiada. Revista de Ciências Médicas e Biológicas, 19(3), 441-448.

Raghoebar, G. M., Meijndert, L., Kalk, W. W., Vissink, A. (2007). Morbidity of mandibular bone harvesting: a comparative study. Int J Oral Maxillofac Implants, 22(3):359-65.

Rodolfo, L. M., Machado, L. G., Betoni-Júnior, W., Faeda, R. S., Queiroz, T. P., \& de SouzaFaloni, A. P. (2017). Substitutos ósseos alógenos e xenógenos comparados ao enxerto autógeno: reações biológicas. Revista Brasileira Multidisciplinar, 20(1), 94-105. 
Research, Society and Development, v. 10, n. 12, e522101220954, 2021

(CC BY 4.0) | ISSN 2525-3409 | DOI: http://dx.doi.org/10.33448/rsd-v10i12.20954

Sampaio, V. P. R., Silva, D. F. B., Barreiro, F. M. P., Brito, H. B. S., de Andrade, F. J. P., \& de Castro Gomes, D. Q. (2020). Implante imediato associado a enxerto xenógeno e provisionalização imediata em área infectada: relato de caso. Archives of Health Investigation, 9(5), 444-448.

Soni, R., Priya, A., Yadav, H., Mishra, N., \& Kumar, L. (2019). Bone augmentation with sticky bone and platelet-rich fibrin by ridge-split technique and nasal floor engagement for immediate loading of dental implant after extracting impacted canine. National journal of maxillofacial surgery, $10(1)$, 98.

Souza, M. T. D., Silva, M. D. D., \& Carvalho, R. D. (2010). Revisão integrativa: o que é e como fazer. Einstein (São Paulo), 8, $102-106$.

Stevenson, S., Emery, S. E., \& Goldberg, V. M. (1996). Factors affecting bone graft incorporation. Clinical Orthopaedics and Related Research®, 324, 66-74.

Troeltzsch, M., Troeltzsch, M., Kauffmann, P., Gruber, R., Brockmeyer, P., Moser, N., Rau, A., Schliephake, H. (2016). Clinical efficacy of grafting materials in alveolar ridge augmentation: A systematic review. J Craniomaxillofac Surg, 44(10):1618-1629.

Urist, M. R., Huo, Y. K., Brownell, A. G., Hohl, W. M., Buyske, J., Lietze, A., ... \& DeLange, R. J. (1984). Purification of bovine bone morphogenetic protein by hydroxyapatite chromatography. Proceedings of the National Academy of Sciences, 81(2), 371-375.

Urban, I. A., \& Monje, A. (2019). Guided Bone Regeneration in Alveolar Bone Reconstruction. Oral and maxillofacial surgery clinics of North America, 31(2), 331-338.

Wang, H. L., \& Carroll, W. J. (2001). Guided bone regeneration using bone grafts and collagen membranes. Quintessence international, 32(7).

Wen, S. C., Huang, W. X., \& Wang, H. L. (2019). Regeneration of Peri-implantitis Infrabony Defects: Report on Three Cases. International Journal of Periodontics \& Restorative Dentistry, 39(5). 